\title{
Effective Use of a Novel Biphasic Calcium Phosphate with Submicron Surface Topography in Posterolateral Spine Fusion
}

\author{
Alwyn Jones* \\ Spire Cardiff Hospital, United Kingdom
}

Submission: March 15, 2021; Published: April 09, 2021

*Corresponding author: Alwyn Jones, MD, Spire Cardiff Hospital, Croescadarn Road Pentwyn, Cardiff CF23 8XL, United Kingdom

\begin{abstract}
The number of spine fusion procedures continues to increase with the aging population. Posterolateral spinal fusion (PLF) is one of the more challenging clinical indications in the spine requiring a bone graft because the aim is to form a large, consolidated bone mass through the paraspinous soft tissues with limited host bone contact. Synthetic calcium phosphate bone graft usage has increased in recent years, reducing the need to harvest large amounts of autologous bone. This class of bone graft closely resembles the composition of human cancellous bone and demonstrates a low incidence of adverse reactions and graft-related complications in the clinic. The body's natural response to the tissue trauma associated with spinal surgery is the upregulation of macrophages of the pro-inflammatory M1 phenotype. This can lead to the formation of scar tissue and failed fusion. This biphasic calcium phosphate (BCP) bone graft with a unique submicron needle-shaped surface topography has been shown in in vitro studies of human-derived monocytes to promote attachment and spreading of anti-inflammatory M2 macrophages, leading to the formation of bone instead of scar tissue. This report on a consecutive case series assessed radiographic, functional and pain outcomes following posterolateral lumbar fusion using a novel biphasic calcium phosphate bone graft with a unique submicron topography in a potentially bone-density-challenged group of female patients.
\end{abstract}

Keywords: Spine fusion; Bone graft; Perimenopausal; Anteroposterior, Lateral radiographs; Biphasic calcium phosphate

\section{Introduction}

The number of spine fusion procedures continues to increase proportionately with the aging population [1,2]. Posterolateral spinal fusion (PLF) is one of the more challenging clinical indications in the spine requiring a bone graft because the aim is to form a large, consolidated bone mass through the paraspinous soft tissues with limited host bone contact. A major obstacle associated with this indication is the lack of available local autograft. As a result, surgeons continue to search for the most advanced and affordable bone graft to achieve successful spine fusion without the well-known co-morbidities related to harvesting autograft [3]. Synthetic calcium phosphate bone graft usage has increased in recent years as it has demonstrated support of bone formation and reduced the need to harvest large amounts of autologous bone $[4,5]$. This class of bone graft closely resembles the composition of human cancellous bone and has proven to be cost-effective with a very low incidence of adverse reactions and graft-related complications [6].
The body's natural response to spinal surgery is the production of macrophages, especially the pro-inflammatory M1 phenotype $[7,8]$. This can lead to the formation of scar tissue and, ultimately, a failed fusion. MagnetOs is a biphasic calcium phosphate (BCP) bone graft with a unique submicron surface topography. Its submicron needle-like surface features have been shown, using in vitro studies of human-derived monocytes, to promote the attachment and spreading of M2 macrophages, leading reliably to the formation of bone instead of scar tissue [9].

In preclinical studies, the BCP has been shown to promote bone formation, even in soft tissue, without the need for added cells or growth factors [10]. This novel BCP is designed to mimic the porous, trabecular structure of cancellous bone. Clinically relevant animal models of posterolateral fusion demonstrate that bone formation take place throughout MagnetOs simultaneously, leading to uniform, solid and stable fusions [11,12]. 
The purpose of this evaluation of a consecutive case series was to assess radiographic success as well as functional and pain outcomes following posterolateral lumbar fusion using a novel biphasic calcium phosphate bone graft with a unique submicron topography (MagnetOs ${ }^{\mathrm{TM}}$, Kuros Biosciences, B.V.).

Four female patients, with an average age at the time of surgery of 62.7 (50-70 years of age), underwent a single or a twolevel posterolateral lumbar fusion procedure for the treatment of degenerative spondylolisthesis or deformity using MagnetOs over the transverse processes and/or the facet joints. Each of these cases involved a perimenopausal/postmenopausal woman; a life stage known to negatively affect bone quality and cause concern when aiming for a successful fusion outcome.

Anteroposterior and lateral radiographs, visual analog scale (VAS) for back and leg pain, the Oswestry Disability Index (ODI), and medication usage were reviewed pre-operatively and post-operatively at 6 and 12 months. VAS and ODI scales were not available at 12 months; however, considering the marked improvement reported by each patient at 6 months, minimal, if any, significant improvement at this time point would be expected at 12 months.

\section{Case Reports}

\section{Case 1: ALIF}

A 69-year-old woman presented with back and left leg radicular pain. Conservative treatment included acupuncture, nerve blocks and physical therapy. Preoperative radiographs and MRI findings revealed lumbar scoliosis and foraminal stenosis at L5-S1. The patient-reported an ODI score of 56 and back pain scores equal to leg pain (8/10) on VAS. Pain was also managed with a combination of Ibuprofen and acetaminophen. Surgical treatment included an anterior interbody/posterior fusion with posterior fixation at L5-S1 and MagnetOs granules mixed with bone harvested from the facets placed laterally to the posterior stabilizing hardware.

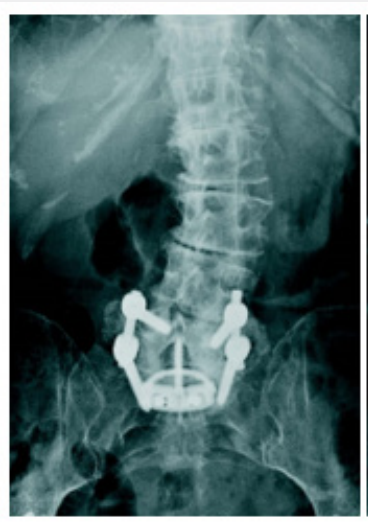

(a)

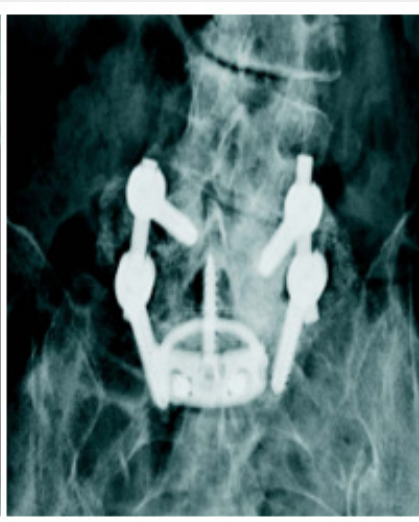

(b)

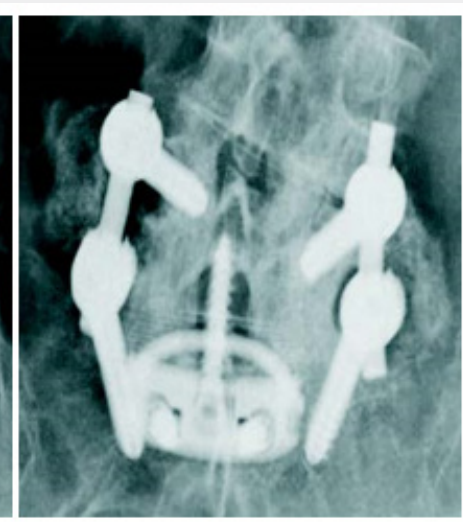

(c)

Figure 1:

a) MagnetOs transitioning from granular appearance to trabecular bone bilaterally at 3 months post-procedure.

b) continuing observable resorption of MagnetOs with good incorporation at 6 months post-procedure.

c) complete fusion mass at 12 months post-procedure.

The patient's leg pain had completely resolved by the 6-month visit. The patient reported completely resolved leg pain, reduced back pain (4/10), an ODI function score of 34 and medication usage had been reduced significantly. Radiographs revealed fusion progression with good incorporation of BCP in the posterior fusion mass. Bone remodeling and graft resorption were evidenced by the loss of granular appearance graft. The 12-month-visit radiographs showed solid bilateral bridging fusion. The patient indicated all pain medication had been discontinued and clinically, no further surgery was indicated (Figure 1).

\section{Case 2: PLIF}

A 50-year-old female presented with intractable 10/10 back pain and 8/10 right leg pain on VAS, pre-operatively. Following failed conservative care, which included MS Contin and Tramadol for pain, nerve blocks and physical therapy, surgery was indicated. Preoperative radiographs and magnetic resonance imaging (MRI) revealed degenerative spondylolisthesis at L4-5. She underwent a posterolateral fusion with instrumentation at L4-5 with MagnetOs granules mixed used as an extender over the lateral gutters combined with a posterior lumbar interbody fusion at L4-5 using a PEEK coated interbody device packed with local bone. Imaging at 6 months showed the progression of fusion with good bone remodeling and the development of bilateral bridging. The patient reported minimal back pain $(3 / 10)$ and leg pain $(1 / 10)$ requiring only Tramadol at $50 \mathrm{mg}$ daily. Her function score dropped from 54 pre-operatively to 20 . At the 12 -month visit, radiographs demonstrated a solid bilateral fusion and Tramadol only as needed (Figure2). 


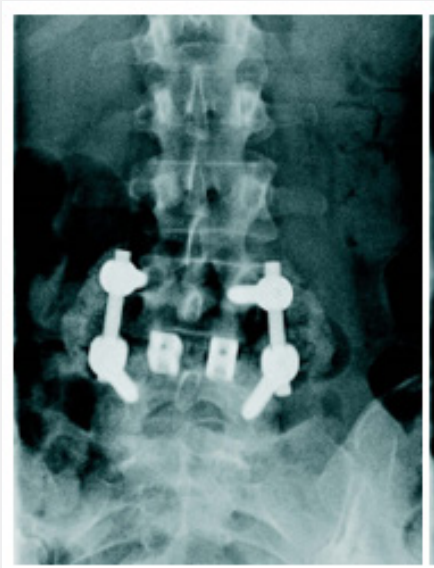

(a)

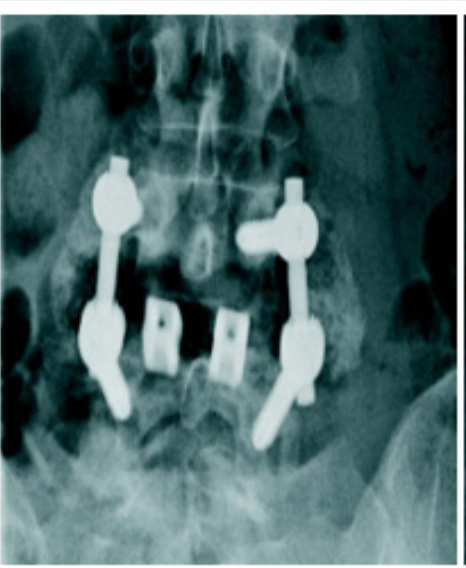

(b)

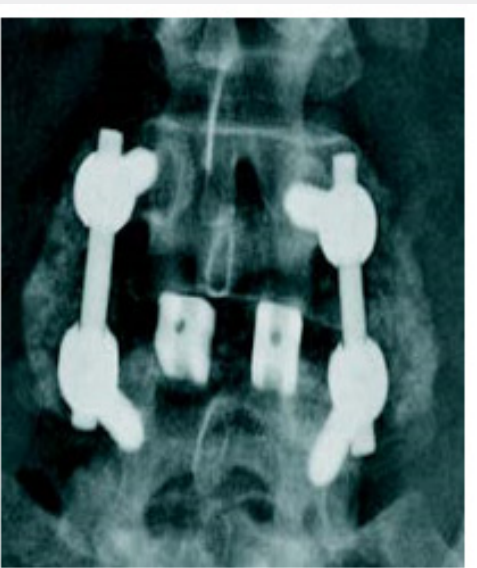

(c)

Figure 2:

a) Evidence of graft material resorption with maturing bone integration at 3 months post-procedure.

b) continued bone remodeling with progression of fusion at 6 months post-procedure.

c) fused; bilateral bridging bone mass observed at 12 months post-procedure.

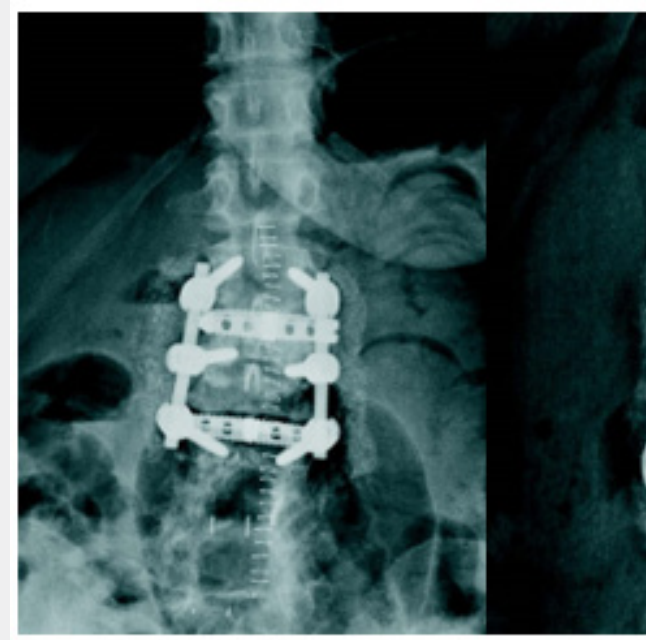

(a)

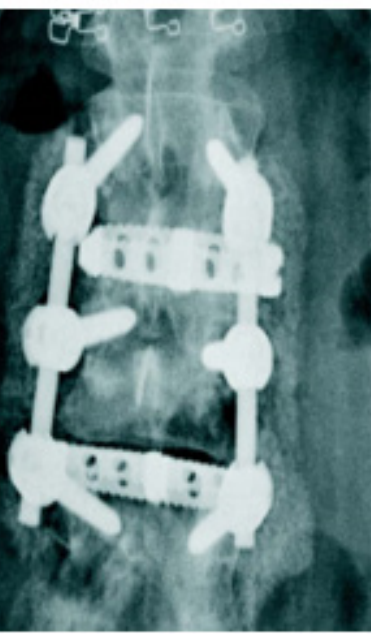

(b)

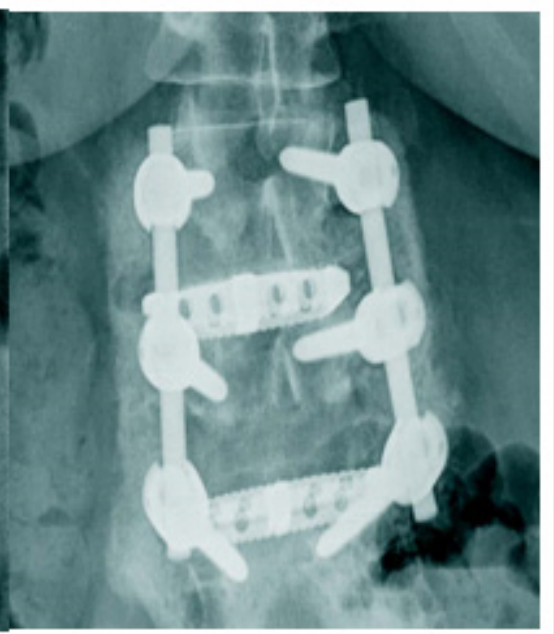

(c)

Figure 3:

a) Early post-operative incorporation with granular appearance at 2 months post-procedure.

b) ongoing remodeling with fusion progression at 6 months post-procedure.

c) complete fusion evidenced by bilateral bridging bone in the lateral gutters at 12 months post-procedure.

\section{Case 3: XLIF}

A 62-year-old female with back pain, bilateral leg pain, and paresthesia. She required the use of a wheelchair to ambulate and has resultant paraspinal muscle atrophy. She had lumbar fusion surgery at L4-5, L5-S1 10 years prior. The patient-reported pre-operative 10/10 back pain, 9/10 leg pain, and a very poor ODI function score of 78 . Conservative care included physical therapy and pain medication through a combination of Duragesic, Duloxetine, and Amitriptyline. MRI and plain radiographs showed degeneration at L2-3, degenerative spondylolisthesis at L3-4, and a fusion L4-S1 with loss of lordosis. The surgical plan involved extending the fusion above the previous fusion levels and decompression at L3-4. A 2-level posterolateral fusion with fixation at L2-3, L3-4 placing MagnetOs granules, which were mixed with local bone, in the inter-transverse process area was performed in 
combination with interbody fusion at the same levels. Radiographs at 6 months demonstrated fusion progression, good incorporation of the BCP into the fusion bed and graft resorption evidenced by the change from granular to more trabecular appearance. The patient was still rehabilitating but reported significantly reduced pain and improved function scores. Her back pain score decreased from $10 / 10$ to $4 / 10$ on the VAS, leg pain decreased from $9 / 10$ to $1 / 10$, and her ODI score improved from 78 to 40 . Pain medication usage reflected the reported improvement in pain, as narcotics had been discontinued. At 12 months, the radiographs revealed a solid fusion bilaterally and medication had been reduced to Tramadol 50mg as needed (Figure 3).

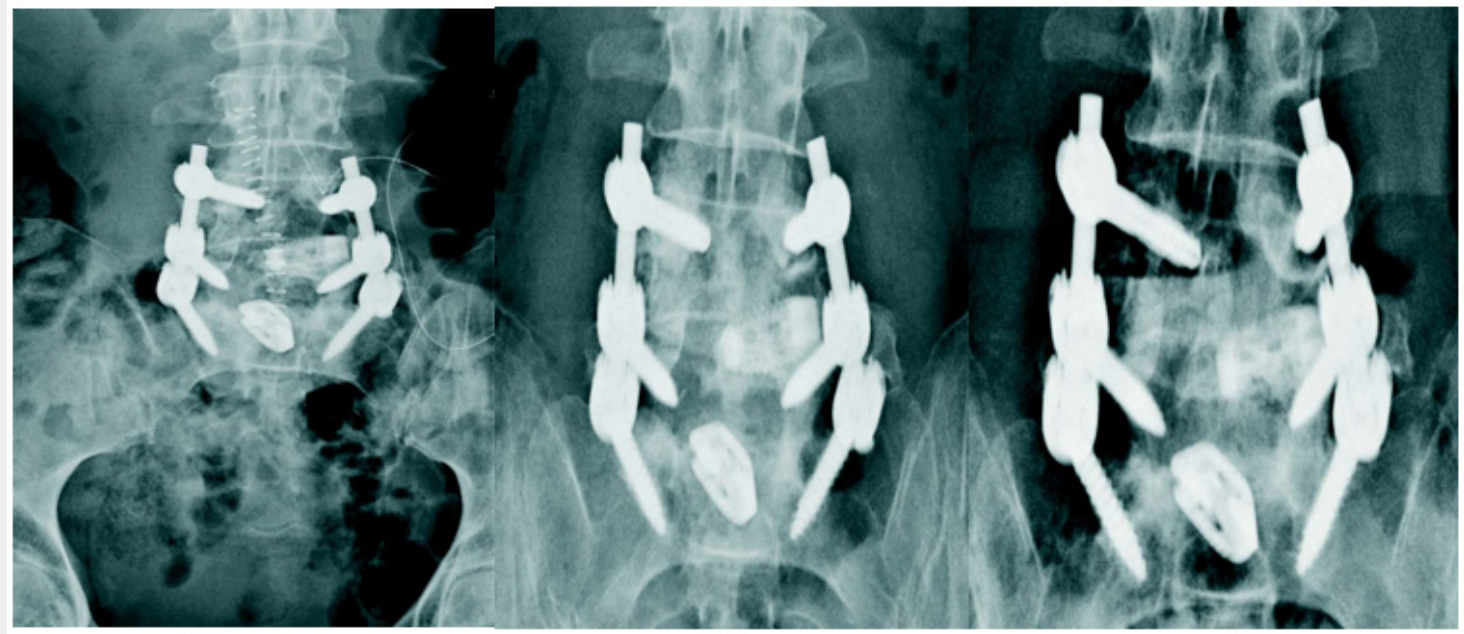

(a)

(b)

(c)

Figure 4:

a) Post-operative radiograph showing MagnetOs over the facets.

b) increased radiopacity over facet joint indicative of fusion progression at 3 months post-procedure.

c) fusion; complete incorporation and transition to solid bone over the facet at 12 months post-procedure.

\section{Case 4: TLIF}

A 70-year-old female with a history of prior decompression at L4-5 and fusion at L5-S1 presented with back pain greater than leg pain. She had not benefited from conservative treatment consisting of physical therapy and pain management. The patientreported $8 / 10$ back pain, 2/10 leg pain, and a poor ODI function score of 60 . Preoperative radiology reports revealed degenerative spondylolisthesis at L4-5. She underwent a posterolateral fusion with fixation at L4-5. BCP granules were mixed with bone harvested from the facets and placed medially to the posterior stabilizing hardware. The surgical plan also included an interbody fusion at L4-5. Radiographic imaging at three months post-op demonstrated fusion progressing well, good graft incorporation and resorption with noticeable changes from granular to a more trabecular appearance. The patient-reported a marked reduction in pain and improved function scores. Her back pain had decreased from $8 / 10$ to $1 / 10$ on the VAS scale, leg pain was completely resolved, and her ODI function score improved by a reduction from 60 to 20. All pain medications had been discontinued other than OTC Tylenol, as needed. At 12 months, the x-rays demonstrated solid fusion and full incorporation of bone graft as demonstrated by increased radiopacity at the facets (Figure 4).

\section{Discussion}

Historically, conventional synthetic bone grafts such as $\beta$-TCP have underperformed when compared to autograft in spinal fusion [13]. The cases described herein clearly demonstrate the effective use of biphasic calcium phosphate with a novel submicron surface topography in achieving fusion in the posterolateral spine. This cohort includes four (4) women of menopausal/post-menopausal age at risk for diminished host bone quality. BCP granules were implanted over the facets and/or in the lateral gutters. It is the posterolateral fusion procedure that presents an additional challenge for fusion, as in this defect, the bone must form over the farthest distance from the host bone.

Each case showed successful outcomes radiographically and in pain and function scores. Regular follow-up x-rays showed the replacement of the granular nature of the graft with trabecular bone over time. Significantly reduced pain and function improvements were demonstrated with a $67.5 \%$ average (range 50\%-90\%) reduction in back pain and a 95\% average (range 90$100 \%$ ) reduction in leg pain. The functional scores improved by an average of 31.5 points (range $22-40$ points) and pain medication usage was dramatically reduced or eliminated. No device-related 
adverse events were reported and none of the patients required revision surgery within 12 months following the index procedure. Despite this being a small cohort of patients, these preliminary findings indicate that this BCP is an effective bone graft for posterolateral fusion, worthy of future clinical research.

This case series review demonstrates successful fusion results in a potentially bone-density-challenged group. It shows that MagnetOs directs bone formation early in the healing process and the graft resorbs at a physiologically balanced rate, thus supporting the transition from woven to trabecular bone and the development of uniform, stable and solid posterolateral fusions. These outcomes confirm results from preclinical studies that the BCP is an ideal bone graft extender for use in posterolateral fusion.

\section{Conflict of Interest}

The study was supported by a grant from Kuros Biosciences $\mathrm{BV}$. AJ is a consultant for Kuros Biosciences BV.

\section{References}

1. Hersey AE, Durand WM, Eltorai A, DePasse JM, Daniels AH (2019) Longer Operative Time in Elderly Patients Undergoing Posterior Lumbar Fusion Is Independently Associated with Increased Complication Rate. Global Spine Journal 9(2): 179-184.

2. Li G, Patil CG, Lad SP, Ho C, Tian W, Boakye M (2008) Effects of age and comorbidities on complication rates and adverse outcomes after lumbar laminectomy in elderly patients. Spine 33(11): 1250-1255

3. Radcliff K, Hwang R, Hilibrand A, Smith HE, Gruskay J, et al. (2012) The effect of iliac crest autograft on the outcome of fusion in the setting of degenerative spondylolisthesis: a subgroup analysis of the Spine Patient Outcomes Research Trial (SPORT). The Journal of bone and joint surgery. American 94(18): 1685-1692.
4. Burger EL, Patel V (2007) Calcium phosphates as bone graft extenders. Orthopedics 30(11): 939-942.

5. Nickoli MS, Hsu WK (2014) Ceramic-based bone grafts as a bone grafts extender for lumbar spine arthrodesis: a systematic review. Global Spine Journal 4(3): 211-216.

6. Holmes RE, Mooney V, Bucholz RW, Tencer AF (1984) A coralline hydroxyapatite bone graft substitute. Clinical Orthopedics and Related Research (188): 252-262.

7. Spiller KL, Nassiri S, Witherel CE, Anfang RR, Ng J, et al. (2015) Sequential delivery of immunomodulatory cytokines to facilitate the M1-to-M2 transition of macrophages and enhance vascularization of bone scaffolds. Biomaterials 37: 194-207.

8. Klopfleisch R (2016) Macrophage reaction against biomaterials in the mouse model - phenotypes, functions and markers. Acta Biomat 43: 3-13.

9. Data on file, 2019

10. Duan R, van Dijk LA, Barbieri D, de Groot F, de Bruijn JD (2019) Accelerated bone formation by biphasic calcium phosphate with a novel sub-micron surface topography. European Cells and Materials 37: 60-73.

11. van Dijk, LA, Duan, R, Luo, X, Barbieri D, Pelletier M, et al. (2018) Biphasic calcium phosphate with submicron surface topography in an Ovine model of instrumented posterolateral spinal fusion. JOR Spine 1(4): e1039.

12. van Dijk LA, Barbieri D, Barrère-de Groot $F$, Yuan H, Oliver R, et al (2019) Efficacy of a synthetic calcium phosphate with submicron surface topography as autograft extender in lapine posterolateral spinal fusion. J Biomed Mater Res B (Part B) 107(6): 2080-2090.

13. Kadam A, Millhouse PW, Kepler CK, Radcliff KE, Fehlings MG, et al. (2016) Bone substitutes and expanders in Spine Surgery: A review of their fusion efficacies. Int J Spine Surg 10: 33.

\section{Your next submission with Juniper Publishers} will reach you the below assets

- Quality Editorial service

- Swift Peer Review

- Reprints availability

- E-prints Service

- Manuscript Podcast for convenient understanding

- Global attainment for your research

- Manuscript accessibility in different formats

( Pdf, E-pub, Full Text, Audio)

- Unceasing customer service

\section{Track the below URL for one-step submission}

https://juniperpublishers.com/online-submission.php 